\title{
Autoantibody Repertoire in APECED Patients Targets Two Distinct Subgroups of Proteins
}

\section{OPEN ACCESS}

Edited by:

Ludger Klein,

Ludwig-Maximilians-Universität München, Germany

Reviewed by: Mitsuru Matsumoto, Tokushima University, Japan Graham Anderson, University of Birmingham, United Kingdom

*Correspondence: Pärt Peterson part.peterson@ut.ee

tPresent address: Mike Rothe, IBA Lifesciences, Goettingen, Germany

\#These authors have contributed equally to this work.

Specialty section:

This article was submitted to Immunological Tolerance and Regulation,

a section of the journal

Frontiers in Immunology

Received: 21 June 2017

Accepted: 31 July 2017

Published: 16 August 2017

Citation:

Fishman D, Kisand K, Hertel C, Rothe M, Remm A, Pihlap M, Adler P,

Vilo J, Peet A, Meloni A,

Podkrajsek KT, Battelino T, Bruserud $\varnothing$, Wolff ASB, Husebye ES,

Kluger N, Krohn K, Ranki A,

Peterson $H$, Hayday $A$ and Peterson P (2017) Autoantibody

Repertoire in APECED

Patients Targets Two Distinct Subgroups of Proteins.

Front. Immunol. 8:976. doi: 10.3389/fimmu.2017.00976

\section{Dmytro Fishman ${ }^{1,2 \ddagger}$, Kai Kisand ${ }^{3 \ddagger}$, Christina Hertel ${ }^{4}$, Mike Rothe ${ }^{4 \dagger}$, Anu Remm ${ }^{3}$, Maire Pihlap ${ }^{3}$, Priit Adler 1,2 , Jaak Vilo ${ }^{1,2}$, Aleksandr Peet ${ }^{5}$, Antonella Meloni ${ }^{6,7}$, Katarina Trebusak Podkrajsek ${ }^{8}$, Tadej Battelino ${ }^{8}$, Øyvind Bruserud ${ }^{9}$, Anette S. B. Wolff ${ }^{9}$, Eystein S. Husebye ${ }^{9}$, Nicolas Kluger ${ }^{10}$, Kai Krohn ${ }^{10}$, Annamari Ranki ${ }^{10}$, Hedi Peterson ${ }^{1,2}$, Adrian Hayday ${ }^{11}$ and Pärt Peterson ${ }^{3 *}$}

\begin{abstract}
'Institute of Computer Science, University of Tartu, Tartu, Estonia, ${ }^{2}$ Quretec Ltd., Tartu, Estonia, ${ }^{3}$ Institute of Biomedical and Translational Medicine, University of Tartu, Tartu, Estonia, ${ }^{4}$ ImmunoQure AG, Düsseldorf, Germany, ${ }^{5}$ Children's Clinic of Tartu University Hospital, Tartu, Estonia, ${ }^{6}$ Pediatric Clinic II, Ospedale Microcitemico, Cagliari, Italy, ${ }^{7}$ Department of Biomedical and Biotechnological Science, University of Cagliari, Cagliari, Italy, ${ }^{8}$ Department of Pediatric Endocrinology, Diabetes and Metabolism, University Children's Hospital, University Medical Centre Ljubljana, Ljubljana, Slovenia, ${ }^{9}$ Department of Clinical Science, University of Bergen, Bergen, Norway, ${ }^{10}$ Department of Dermatology, Allergology and Venereology, Institute of Clinical Medicine, University of Helsinki, Skin and Allergy Hospital, Helsinki University Central Hospital, Helsinki, Finland, ${ }^{11}$ Peter Gorer Department of Immunobiology, King's College, Guy's Hospital, London, United Kingdom
\end{abstract}

High titer autoantibodies produced by B lymphocytes are clinically important features of many common autoimmune diseases. APECED patients with deficient autoimmune regulator (AIRE) gene collectively display a broad repertoire of high titer autoantibodies, including some which are pathognomonic for major autoimmune diseases. AIRE deficiency severely reduces thymic expression of gene-products ordinarily restricted to discrete peripheral tissues, and developing $T$ cells reactive to those gene-products are not inactivated during their development. However, the extent of the autoantibody repertoire in APECED and its relation to thymic expression of self-antigens are unclear. We here undertook a broad protein array approach to assess autoantibody repertoire in APECED patients. Our results show that in addition to shared autoantigen reactivities, APECED patients display high inter-individual variation in their autoantigen profiles, which collectively are enriched in evolutionarily conserved, cytosolic and nuclear phosphoproteins. The APECED autoantigens have two major origins; proteins expressed in thymic medullary epithelial cells and proteins expressed in lymphoid cells. These findings support the hypothesis that specific protein properties strongly contribute to the etiology of B cell autoimmunity.

Keywords: autoimmune regulator, autoantibodies, immune tolerance, thymus, autoantigen

\section{INTRODUCTION}

Many severe multifactorial autoimmune diseases are in part defined by pathognomonic antibodies that provide important clinical tests of disease predisposition or status. However, whereas our knowledge of the genetic and cellular factors contributing to multifactorial autoimmune diseases is increasing, we remain largely ignorant of properties of autoantigens and why only selected proteins are targeted in autoimmunity (1). To investigate B cell autoantibody repertoire, we have examined APECED (autoimmune polyendocrinopathy with candidiasis and ectodermal dysplasia) patients (2), defined by rare monogenic defects in the autoimmune regulator (AIRE) gene that drives the expression of tissue-restricted self-antigens in medullary thymic epithelial cells (mTEC) (3-5). T cell 
progenitors with strong reactivity toward such self-antigens are eliminated or functionally inactivated. Lacking AIRE function, APECED patients predictably accumulate many autoreactive T cells, but in addition they collectively display high titer autoantibodies to multiple self-proteins (2).

The antibodies with the highest reported titers target type I interferons (IFNs) and have become diagnostic or even prognostic markers for APECED (6). Neutralizing type I IFN antibodies mostly appear early in life (7), inhibit IFN-stimulated gene expression (8), and were recently shown to correlate inversely with the onset of type 1 diabetes (T1D) in APECED (9). A second group of APECED-associated autoantibodies targets Th17-associated cytokines, IL17A, IL17F, and IL22, emerges early in the disease course (7), and is associated with chronic mucocutaneous candidiasis $(10,11)$, another defining feature of APECED. Interestingly, our in-depth studies of anti-cytokine antibodies harbored by APECED patients showed that the functionally rearranged immunoglobulin gene sequences were heavily mutated, encoding antibodies of extremely high affinity that we hypothesized to result from B cell dysregulation in germinal center reactions (9). Such etiology can be contrasted with the amplification and maturation of natural, polyreactive autoantibodies that are normally of relatively low affinity, which has been proposed to underpin sporadic autoreactivity in otherwise healthy individuals (12).

There is a prominent set of APECED-associated autoantibodies that are shared with multifactorial autoimmune diseases, such as those directed against glutamic acid decarboxylase (GAD)1 and GAD2 in T1D (13), or against CYP21A2 in Addison's disease (14). Therefore, the comprehensive analysis of autoantibodies in APECED may offer improved insight into the etiology of such diseases. These autoantibodies have often been identified by candidate or cDNA expression library screenings. However, proteome arrays provide unprecedentedly broad coverage to identify novel target proteins, such as melanoma MAGEB antigens and testis-specific PDILT, which were recently identified as autoantigens in APECED (15).

To gain further insight, we searched common features of autoantigenic proteins by a systematic analysis of a protein microarray platform, which allows large-scale, unbiased screening of autoantibody reactivities. Herein, we report a broad spectrum of autoantigens that is collectively targeted by 82 APECED patients. This APECED "autoimmunome" is not a random collection of proteins but comprises two sub-groups, those whose expression is most likely AIRE-regulated in the thymus and those enriched in lymphoid tissue. Moreover, these autoantigens are enriched in evolutionarily conserved cytosolic and nuclear proteins with high propensity for post-translational modifications. Reactivity to an increased number of such autoantigens was a stronger correlate of clinical symptoms than was either patient age or time since disease onset.

\section{MATERIALS AND METHODS}

\section{Patient Samples}

Altogether 100 sera samples from 82 individual patients were profiled in Protoarray, as described (9). For longitudinal analysis, we collected 2 samples from 11 patients; 3 samples from 2 patients; and 4 samples from 1 APECED patient. Only one serum sample (with maximal number of positive hits) per patient was used in all analyses, except in longitudinal analysis where multiple samples taken at different time points were available from 14 patients. The patients were from Finland, Italy, Norway, Slovenia, and Estonia and diagnosed according to mutational analysis of the AIRE gene and by the presence of autoantibodies to type I IFNs. The mean age of the patients was 31 years. The main characteristics of the patients are given in Table S1 in Supplementary Material. The control group consisted of 20 individuals (12 healthy volunteers and 8 healthy first-degree relatives of the Italian APECED patient cohort). The study was carried out in accordance with the recommendations of local ethics committees (Finland: HUS Medical ERB, 8/13/03/01/2009; Slovenia: National Medical Ethics Committee number 22/09/09 and 28/02/13; Italy: Ethics Committee Prot. PG/2015/20440; Norway: Research Ethics Committee of Western Norway, health registry number 047.96, bio-bank number 2013-1504, project number 2012/1850; Estonia: Research Ethics Committee of the University of Tartu, 235/M-23) with written informed consent from all subjects, as described earlier (9). All subjects gave written informed consent in accordance with the Declaration of Helsinki.

\section{Protein Array Screening}

The autoantibody screening was performed by ISO9001 certified Custom ProtoArray Service Lab at Invitrogen (Thermo Fisher Scientific). Briefly, the protein arrays (ProtoArray v5.1) were probed as described in Invitrogen's protocol for Immune Response BioMarker Profiling using detection reagent (Alexa Fluor 647 Goat Anti-Human IgG A21445, Invitrogen) and blocking buffer (Blocking Buffer Kit PA055, Invitrogen). Arrays were scanned using a GenePix 4000B fluorescent scanner, and the data were acquired with GenePix ${ }^{\circledR}$ Pro software. The arrays were probed with sera at a dilution of 1:500.

\section{Data Cleaning, Normalization, and Print Contamination}

Previous studies involving protein microarrays (16) have shown that raw intensity values should not be compared directly because of technical, physical, chemical, and individual variability, occurring mostly at the production stage. In order to preserve true biological signal and concurrently eliminate potential technical noise, we applied a robust linear model (RLM) approach (17), using $r l m$ function in R. RLM is considered to be the state of the art normalization technique for protein microarray data (18) and is applied on control probes that are assumed to have constant positive signal across all arrays. We used human-IgG and V5 control series for normalization as reported (17). Background subtracted signal was log-transformed prior to applying RLM to approximate for normal distribution (Figure S1 in Supplementary Material). To address printing contamination, we excluded all autoantibody targets that showed high correlation $(>0.6)$ with previously reported autoantigens. In addition, we identified and eliminated highly correlated proteins located in the neighboring wells on the array. In total, we identified and removed 31 false positives, some of which were prevalently positive across many samples (Figure S2 in Supplementary Material). The normalized signal was standardized using mean and SD of healthy samples 
(including healthy heterozygous relatives). Proteins, with standardized signal ( $z$-score) above 3 in three or more patients were regarded as autoantigens. The full list of the positive proteins is provided in Table S2 in Supplementary Material.

\section{Differential Data Analysis and Clustering of Autoantibody Reactions}

In order to identify the proteins correlating with various clinical manifestations, we used moderated t-statistics implemented in ebayes function from limma $\mathrm{R}$ package (19) on normalized intensities from all 9,000 proteins. Obtained $p$-values were corrected using false discovery rate correction method and top significant proteins (corrected $p$-value $<0.05$ ) were extracted for each manifestation resulting in six significant associations. Unsupervised hierarchical clustering using pheatmap package in $\mathrm{R}$, which internally uses hclust function for clustering rows and columns of matrix. Linear regression analysis of dependency between number of positive hits and number of manifestation was analyzed with $l m$ method in R.

\section{Variability of APECED Autoantigen Profile over Time}

We extracted data for 14 patients with multiple samples and formed all possible pairs of samples that belong to the same patients and divided these pairs into two broad categories: pairs of samples that were obtained $>10$ years apart (eight samples, on average 24.4 years apart) and samples that were obtained $<10$ years apart (13 samples, on average 1.5 years apart). Reactive proteins were compared between samples of the same patient and the percentage of the proteins that were specific to early, late, or shared between samples for both of the categories was computed. As expected, samples taken close in time had more reactive proteins in common, then samples obtained more than 10 years apart. Samples taken more than 10 years apart show higher percentage of late sample specific proteins, which might mean that with time repertoire of reactive proteins gets larger.

\section{Correlation with APECED Mutations}

We divided the patients into three groups based on their mutations: (1) homozygous for p.R139X, (2) homozygous for p.R257X, and (3) homozygous p.L323fsX373 or compound heterozygotes of either p.L323fsX373 or missense mutations. We used pairwise comparisons of Tukey and Kramer (Nemenyi) test with TukeyDist approximation for independent samples.

\section{The Enrichment Analyses for Protein Characteristics}

To assess intrinsic features of positive reactivities, we used data from the following public databases: Human Protein Atlas (20), Compartments DB (21), dbPTM (22), UniProt Knowledgebase (23), Ensembl (24), OrthoDB8 (25), and SUPERFAMILY (26). We used gconvert function from gProfileR package in R (27) to convert names of genes and proteins into Ensembl gene identifiers (ENSG). Size of the overlap between our group of positive proteins and every set extracted from each database was estimated. Using hypergeometric test for each overlap, we computed probability values to estimate how likely it was to observe this overlap or larger by random chance. We used false discovery rate procedure to adjust obtained $p$-values and significance threshold of 0.05 to reject a null-hypothesis. To double check our findings, we used g:Profiler web-tool (27) by submitting our list of positive proteins and running unordered query with ProtoArray content as a background to account for any design bias.

\section{Single Nucleotide Polymorphism (SNP) and Evolutionary Conservation Analysis}

For SNP analysis, we retrieved all SNPs associated with each gene on our platform that was found in Ensembl database (7,284 genes). To retrieve this information, we used $\mathrm{R}$ command useMart and the following parameters: useMart("ENSEMBL_ MART_ENSEMBL", dataset = 'hsapiens_gene_ensembl', host $=$ "www.ensembl.org"). We then calculated the number of SNPs within boundaries of the gene and SNPs that are located at the exon regions of the gene. We normalized these values by associated gene length (distance between two furthest associated SNPs), by gene length, and by accumulative length exon regions of each gene, respectively. To compare positive proteins with the rest of platform in terms SNP counts, we first sampled 10,000 random groups of genes of the same size as our group of reactive proteins. For each group, we computed mean counts of two categories. For evolutionary conservation analysis, the data were obtained from ftp://cegg.unige.ch/OrthoDB8/ Eukaryotes/. We downloaded four pairs of genes and annotations for all_species, mammalia, metazoa, and vertebrata. In all four gene files, we filtered out genes not related to Homo sapiens and converted Ensemble protein IDs into gene IDs using biodbnet (http://biodbnet.abcc.ncifcrf.gov/db/db2db. $\mathrm{php}$ ) software. We calculated an average evolution rate for our group of reactive proteins (for all genes that had evolutionary rate recorded in $\mathrm{DB}$ ). Then we sampled 10,000 random groups of the same size as our reactive group and calculated an average evolutionary rate.

\section{Luciferase Immunoprecipitation (LIPS) Analysis}

Luciferase immunoprecipitation assay was performed as reported $(28,29)$. Autoantigen cDNAs were cloned into modified $\mathrm{pNa}$ noluc vector (Promega, Madison, WI, USA) downstream of naturally secreted NanoLuc luciferase (Nluc). HEK293 cells were transfected with the plasmid constructs and cell culture medium containing Nluc-fusion proteins was collected after $48 \mathrm{~h}$. Serum samples were first incubated with the fusion protein solutions overnight, then Protein $\mathrm{G}$ agarose beads were added and incubated at room temperature for $1 \mathrm{~h}$ in 96-well microfilter plates (Merck Millipore, Billerica, MA, USA) to capture antibodies and immune complexes to the beads. After the washing to remove unbound fusion proteins, a luciferase substrate (furimazine) was added to reaction, and luminescence intensity (LU) was measured in Victor X Multilabel Plate Reader (PerkinElmer Life Sciences, Waltham, MA, USA). Results were expressed as relative units LU sample/average LU of healthy control samples. The positive/ 
negative discrimination level was set to the mean plus three SDs of the healthy control samples.

\section{RESULTS}

\section{Protoarray Screening of APECED Sera}

The basis for our unbiased study of APECED autoreactivities was a protein microarray (Protoarray) of approximately 9,000 proteins printed to a single glass slide. Against this array, we screened 100 sera from 82 patients of Finnish, Norwegian, Slovenian, Sardinian, and Estonian origins, together with control sera obtained from healthy heterozygous relatives $(n=8)$ and unrelated healthy controls $(n=12)$ across the same age range.

The arrays were normalized by RLM (17) based on control protein intensities with further standardization and limitation of false-positives (described in Materials and Methods). The positive proteins were defined as signals with a z-score (the number of standard deviations SDs from the mean of combined controls) of greater than 3 (which is more stringent than the common practice of calling hits at $z>2$ ). Although APECED patients altogether displayed significantly different set of autoreactivities in comparison to controls, the reactivity profiles contained multiple patient-specific reactivities in addition to shared autoantibodies (9). Therefore, to intensify the likely biological and clinical significance of hits, we considered a protein to be an autoantigen only when it was recognized by $\geq 3$ individual patient samples (see Materials and Methods). When this additional stringency was applied, proteins encoded by 963 unique Refseq genes qualified as autoantigens.

\section{Autoantigen Repertoire Contains Shared and Individual Reactivities}

As expected, multiple sera displayed strong reactivities toward approximately 20 proteins that were previously reported as APECED autoantigens, including type I IFNs (Figure 1A), Th17 cytokines, GAD1, GAD2, DDC, TGM4, GIF, HSD3B2, BPIFB1, TSGA10, CYP1A2, KCNRG, and AQP2 (Figure 1B). Moreover, most such reactivities were also detected by LIPS assay (Figure S1 in Supplementary Material). It should be noted that apart of HSD3B2, the set of proteins on Protoarray did not include the steroidogenic enzymes CYP21A2, CYP17A1, and CYP11A1, which have been demonstrated as autoantigens in APECED.

Among shared reactivities, the highest titers were shown by interferon- $\alpha$ (IFNA)-specific antibodies, for which we identified some strong correlations (coefficients from 0.41 to 0.98 ), the highest being between IFNA2 and IFNA4 (correlation 0.98), and IFNA6 and IFNA17 (correlation $~ 0.93$ ) (Figure 1C). These correlations could not be explained by the levels of primary sequence similarity or by close phylogenetic relationships (Figure S2 in Supplementary Material), and are instead likely to reflect shared conformational epitopes in IFNA proteins. Likewise, there was commonly a strong correlation (coefficient from 0.41 to 0.62 ) of IFNA reactivity with reactivity toward IFN- $\omega$ (IFNW) (Figure 1C).

In addition to shared reactivities, we found high interindividual variation in the number and identity of autoantigens
(Figure 1D). To analyze a covariance of sample groups, we performed a principal component analysis, which showed that the relatively homogenous group of combined healthy relatives and control samples readily segregated from the patient samples that displayed a large intra-group variability (Figure 1E).

\section{Multiple Specificities Include Cancer- Testis and Testis-Specific Autoantigens}

Among the strong, "rare" specificities, there was a number of potential autoantigens depicted in Figure 2A. Of these, LCN1 was reported as an autoantigen in Sjögren syndrome patients (30), and HMGB1 was reported to be associated with the production of anti-DNA autoantibodies in systemic lupus erythematosus (31). To extend the Protoarray results, we used LIPS to assay reactivity toward eight of these proteins (LCN1, MKNK2, POMZP3, BAALC, FGF12, HMGB1, RPL12, and S100A7A) of sera from a subgroup of 30 Finnish APECED patients (Figure 2B). The first three showed particularly strong positive correlations between Protoarray and LIPS results (Figure 2C), but given that the reactivities were often rare and that the patient groups tested in the two assays did not completely overlap, it was not surprising that correlations were not always statistically significant. Of note, however, this did not reflect a general tendency of the Protoarray to reveal false positives, since some sera showed much stronger LIPS reactivity to defined targets (e.g., RPL12, FGF12, HMGB1; Figure 2C). Indeed, this may reflect the potential of the Protoarray to underestimate reactivities of conformation-specific autoantibodies, because of the denatured states of many of the arrayed proteins and protein fragments.

Interestingly, we noted reactivities toward $\sim 20$ so-called "cancer-testis autoantigens" (CT-As) (Figure 2D). CT-As can be expressed in a wide variety of malignant tumors, where they are known to be immunogenic, but their expression in normal tissue is mostly restricted to germ cells in testis, fetal ovary, and placenta (32). The extent of reactivity revealed by our screen far exceeded the previous report of shared APECED reactivity to CT-As PDILT and MAGEB2 (Figure 2D) in a large-scale screening of another APECED cohort (15). Rather, we found reactivities toward several proteins from the MAGE-AMAGE-B family that are expressed in melanoma and other tumor types but which in normal tissues are expressed only in the testis; reactivities toward GAGE1 and GAGE7B, which are members of another X-chromosome linked, CT-A family; and reactivities toward SPAG8 and SPAG16 (Figure 2D). The latter two in particular highlight the cross-over of APECED serum autoreactivities with other pathophysiologies, in that SPAG8 was initially identified as a sperm-associated antigen target of serum of an infertile woman (33), while SPAG16 is expressed in sperm and in reactive astrocytes of lesions in multiple sclerosis patients in whom it has been identified as an autoantibody target (34). Of note, both males and females showed autoreactivities toward several sperm-specific proteins (Figure 2E). Protoarray reactivities toward 11 testis specific and CT-antigens tested showed good overall correlation with LIPS (Figures 2E,F). 

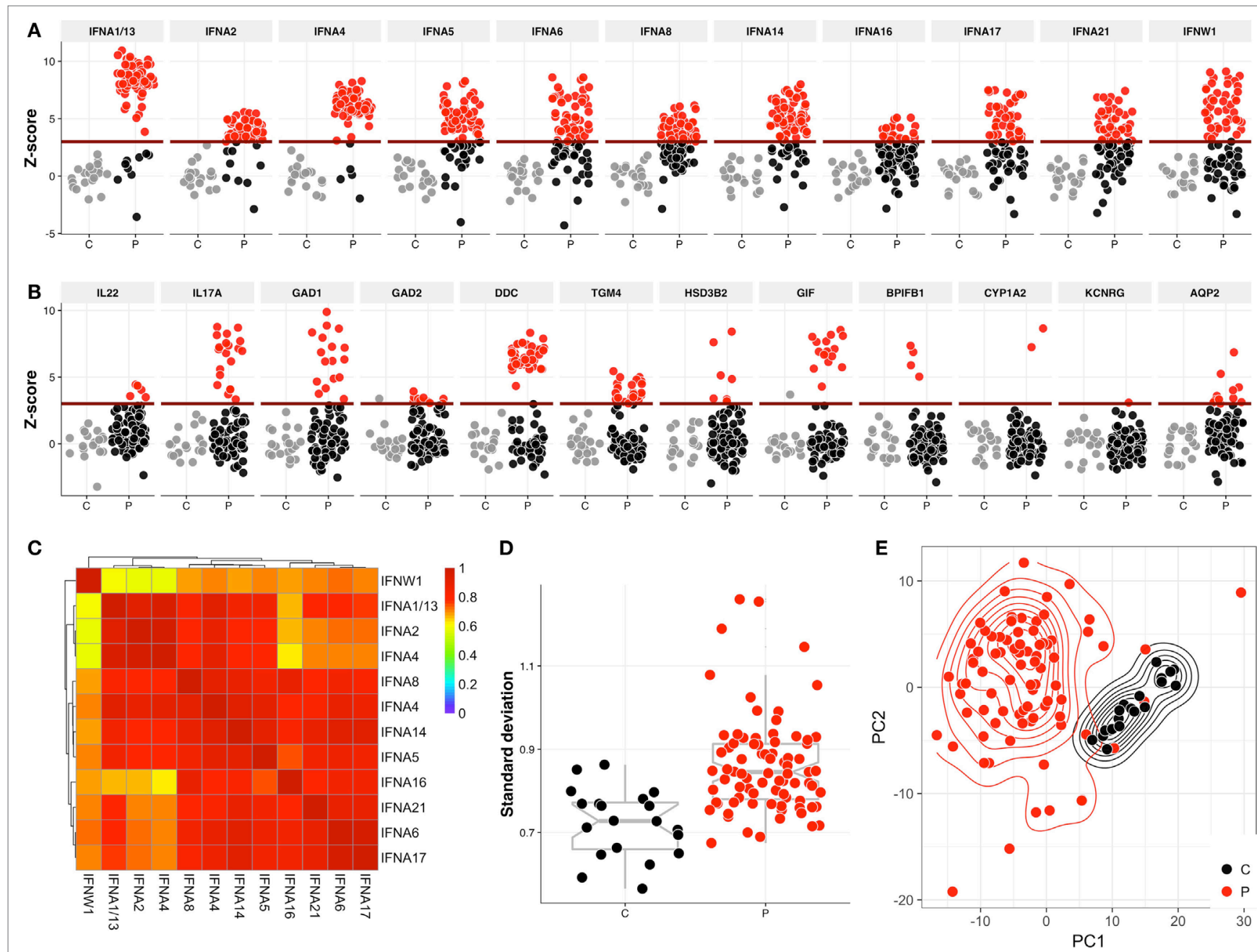

FIGURE 1 | Protoarray reactivities to type I interferons (IFNs) and to known autoantigens. The reactivities to (A) type I IFNs and (B) known APECED autoantigens. The Protoarray signals are expressed as $z$-scores representing the number of SDs from the mean of combined control samples. Positive-negative discrimination level (dark red line) was set at $z=3$. Red circles represent the samples with $z>3$, gray circles are control samples. (C) Heatmap of correlation coefficients of type I IFN reactivities between each other. (D) Variation analysis of controls and patients-each dot on this figure represents a SD within sample over all Protoarray signals. Red circles represent patients $(\mathrm{P})$ and black controls $(C)$. Variance in patient samples are significantly higher $(p=2.598 \mathrm{e}-07)$ in comparison to control samples. (E) Principal component analysis plot of controls and patients. The first two principle components explain 35.8 and $11.5 \%$ of total variance, respectively. First 50 most common autoantibody reactivities were used in order to build this plot.

\section{Autoantibody Correlations with Clinical Manifestations}

We next analyzed whether immunoreactivities to certain autoantigens correlated with disease components of individual APECED patients (Figure 3). Pernicious anemia correlated with seven reactivities, including gastrointestinal factor GIF that is an established autoantigen in pernicious anemia patients (35); five specificities, including GAD1 and GAD2, correlated with vitiligo; and autoantibodies to GABPB2 correlated with autoimmune hepatitis in APECED patients. By contrast, several associations described previously among small-scale studies, e.g., DDC with $\mathrm{AIH}$ and vitiligo $(36,37)$, were not revealed. In addition, we confirmed that reactivity toward prostate specific antigen, TGM4, was almost restricted to post-pubertal males (Figure S3 in Supplementary Material) (38).
To test whether the autoantibody reactivities might segregate APECED patients into subgroups, we performed unsupervised hierarchical clustering of patients and the 50 most-reactive autoantigens, from which we excluded type I IFNs. This analysis clearly revealed two patient sub-groups with overall low and high reactivity repertoires, respectively (Figure 4A). An overlay of this heatmap with clinical data showed that the number of autoantigens targeted was positively correlated with the number of clinical manifestations, as confirmed by linear regression (Figures 4B,C).

As the number of disease components in APECED patients is known to increase over time, we tested whether the number of autoantibody targets in each patient is likewise related to the age of onset of the first clinical manifestation. However, the trend toward correlation between the number of diseases and either age or time since diagnosis was not significant (Figure S4 

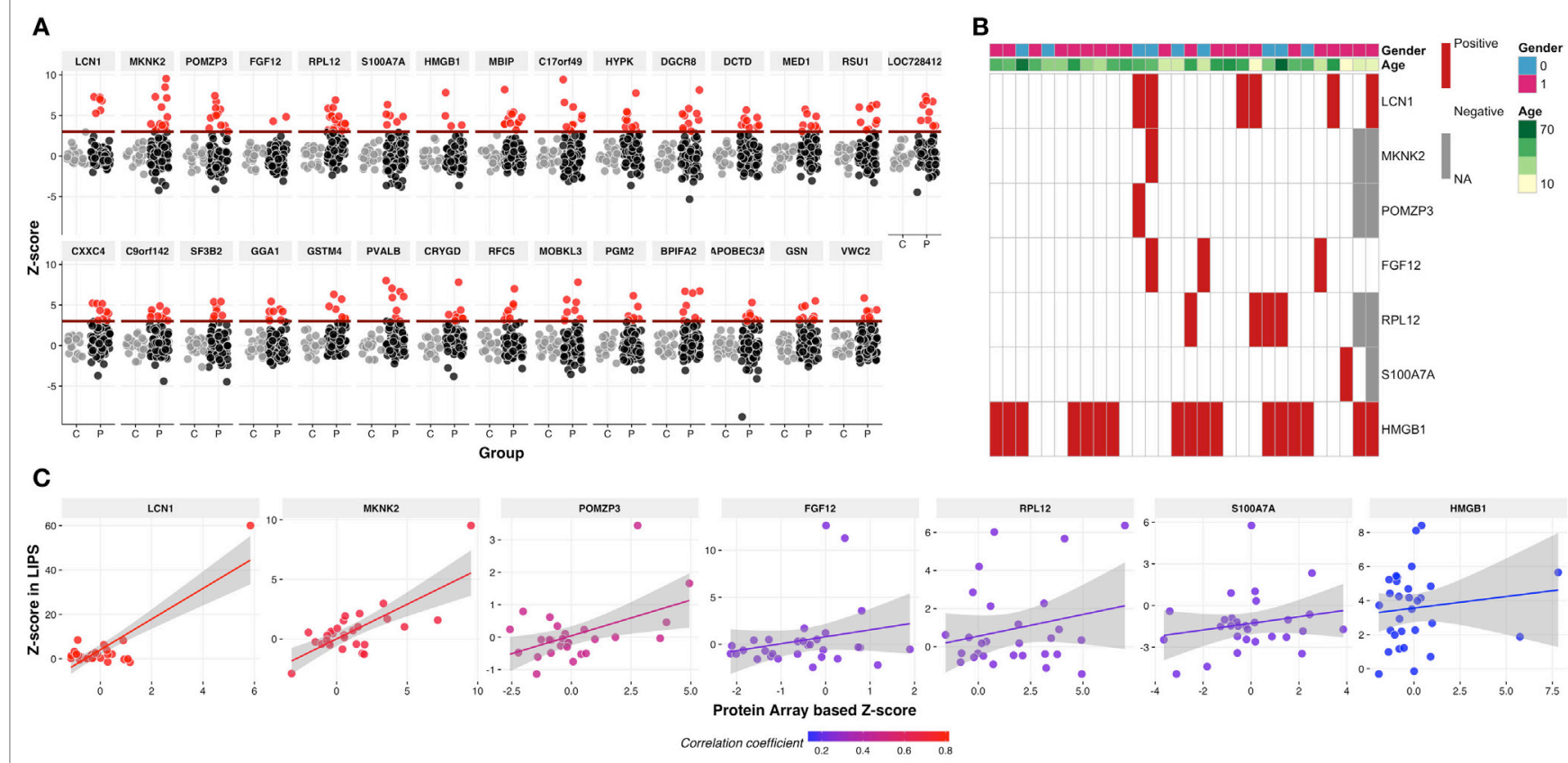

D

E
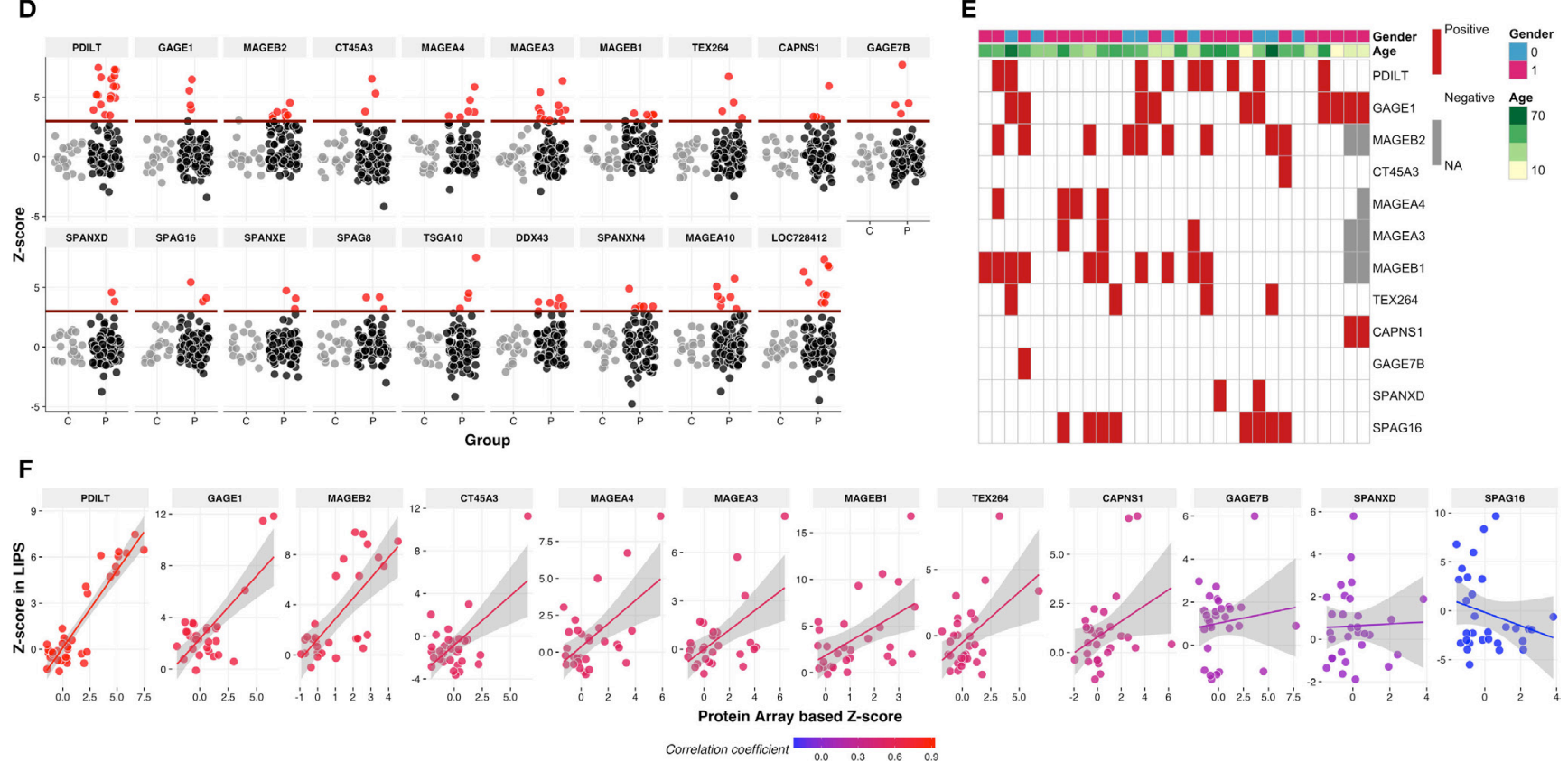

FIGURE 2 | Autoantibody reactivities to selected novel autoantigens. (A) The Protoarray signals are expressed as z-scores representing the number of SDs from the mean of combined control samples. Positive-negative discrimination level (dark red line) was set at $z=3$. Red circles represent the samples with $z>3$, gray circles are control samples. (B) Seven out of the 29 novel autoantigens were tested in luciferase immunoprecipitation (LIPS) using a subset of 30 Finnish APECED patients' samples. Red color in the cells of the heatmap represents positive reactivity $(z>3)$, gray cells indicate missing values and white color stands for missing autoantibodies. Rows on top indicate gender and age. (C) Correlation analysis of Protoarray and LIPS results on the autoantigens in panel (B). (D) z-Scores to 19 cancer-testis antigens (CT-As) in APECED patients according to Protoarray results coded as in panel (A). (E) Twelve of identified CT-As were retested using LIPS assay in a subset of 30 Finnish APECED patients. Color code as in panel (B). (F) Correlation analysis of Protoarray and LIPS results of CT-As in panel (E). Color of dots and fitted linear trends in panels (C,F) represent the value of correlation coefficient, higher coefficient values are encoded as red color and low or negative correlation as blue.

in Supplementary Material), arguing that neither can independently explain the accumulation of multiple clinical entities in APECED. Instead, these findings add further weight to the conclusion that the complexity of the autoantibody response is a major underlying factor for the expansion of clinical profile in APECED.

Previous studies have shown changes in autoantibody profiles over time. To understand the dynamics of autoantibody 

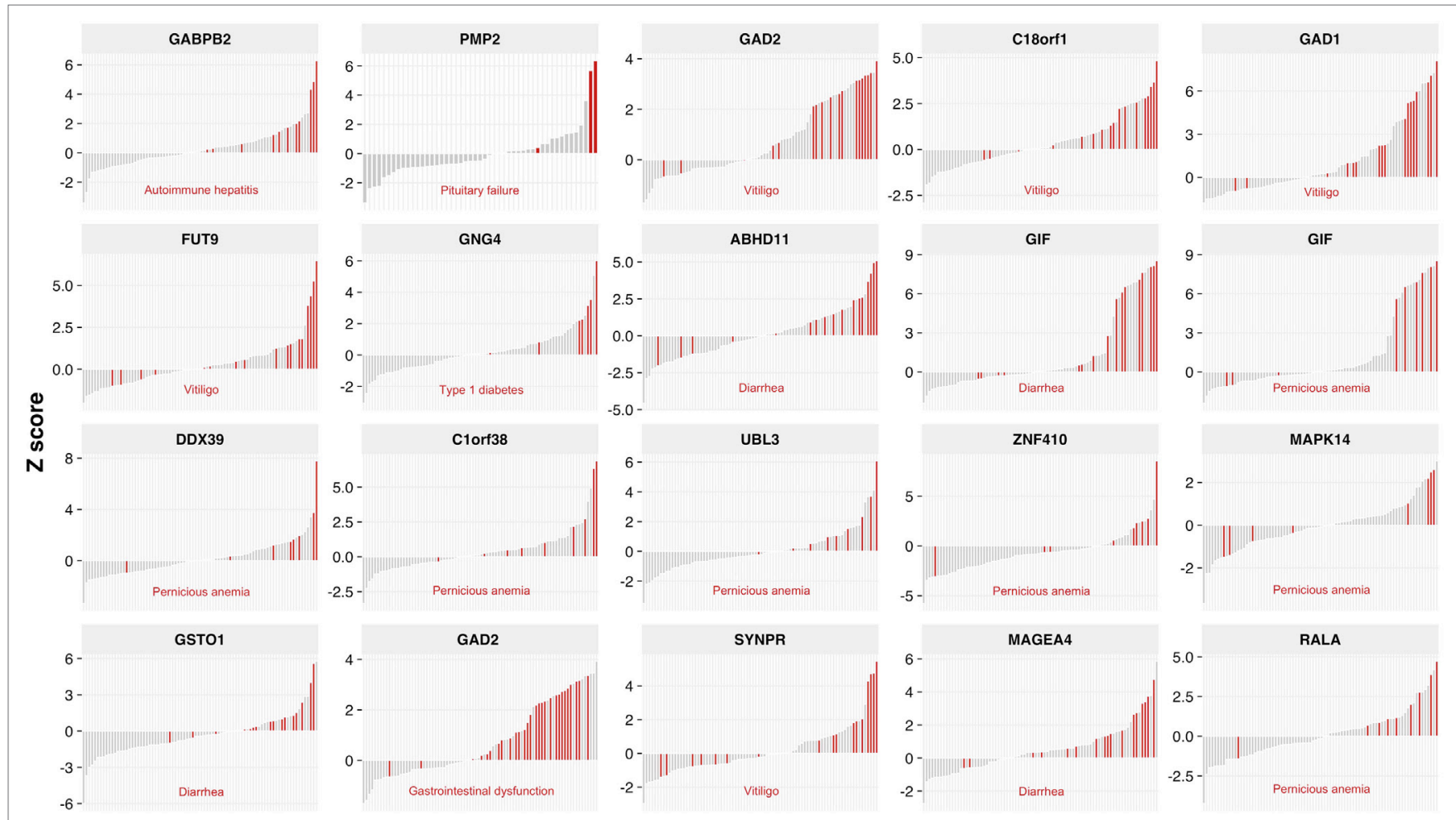

Array

FIGURE 3 | Autoantibody reactivities associated with APECED patients clinical and phenotypic features. Association of Protoarray z-scores with corresponding clinical manifestations. The height of the bar depicts $z$-score of the sample, red bars represent samples positive for a given manifestation (indicated below each plot). All other bars in gray represent autoantibody levels in patient samples without the certain clinical manifestation. Only statistically significant associations are shown $(p<0.05)$ and adjusted across 900 most reactive proteins on Protoarray using moderated contrast $t$-test.

repertoire, we studied 14 APECED patients whose sera were collected at least twice in different time points and, thus, analyzed longitudinally by Protoarray. In most patients $(n=11)$, the number of proteins recognized by autoantibodies increased with time (Figure 4D), whereas in only three patients the number of reactivities decreased or remained almost unchanged. We next studied whether the target antigen profile in APECED patients broadens over time. For this, we compared the samples taken less than 10 years apart with the samples spanning more than 10 years in three separate categories: (i) target specific to early sample, (ii) target shared in early and late sample, and (iii) target specific to late sample. Expectedly, the proportion of targets specific to late sample was larger indicating that the samples taken closer in time shared more reactivity to autoantigens than samples obtained more than 10 years apart (Figure 4E).

\section{Nature of APECED Autoantigens}

Of the many AIRE truncating mutations identified in APECED patients, the most prevalent are p.R257X creating a premature stop codon in exon 6, and a 13bp deletion, p.L323fsX373, changing the reading frame and causing a premature stop codon in exon 8. Although both mutations are present in APECED patients with various ethnic backgrounds, the R257X is commonly found among Finnish (83\% of APECED alleles), Norwegian and Slovenian patients. By contrast, the earliest
AIRE truncation occurs in Sardinian patients who share a p.R139X mutation that introduces a premature stop codon prior

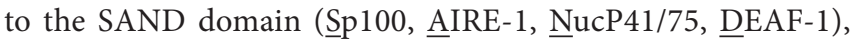
an $\sim 80$ residue region common to many chromatin-regulating factors. When we divided the patients into three groups based on these genetic etiologies [(1) homozygous for p.R139, (2) homozygous for p.R257X, and (3) homozygous p.L323fsX373, or compound heterozygotes of either p.L323fsX 373 or missense mutations], we found patients in Groups 1 and 2 to react to a wider spectrum of autoantigens (Figure 5A), thereby correlating autoantibody reactivities with the p.R139X and p.R257X truncation mutations.

Intrinsic features of proteins that might promote their autoantigenicity are not known. Indeed, the available information on the repertoire of autoantibody target proteins is fragmented, and based on this, few if any overt similarities have emerged among proteins identified as autoantigens. Given the scale of the analysis described here, we systematically analyzed our list of autoantibody target proteins for any parameters that might be enriched relative to proteins that were not targets (Figure 5B; Table S2 in Supplementary Material).

We first studied the subcellular localizations of APECED autoantigens by employing two different approaches: (i) the Compartments resource that integrates sequence-based and manually curated subcellular localization information from 


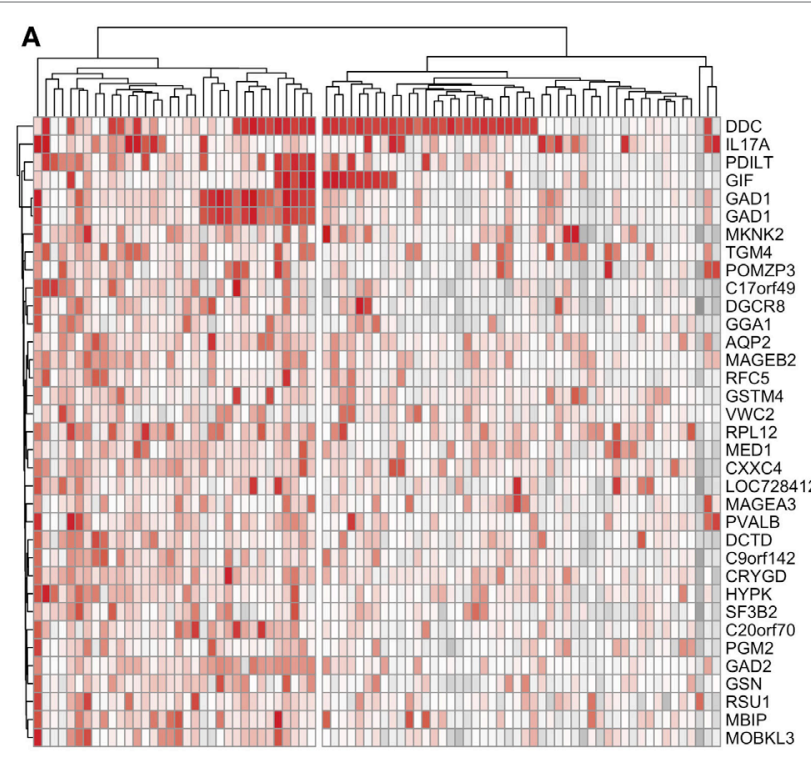

C

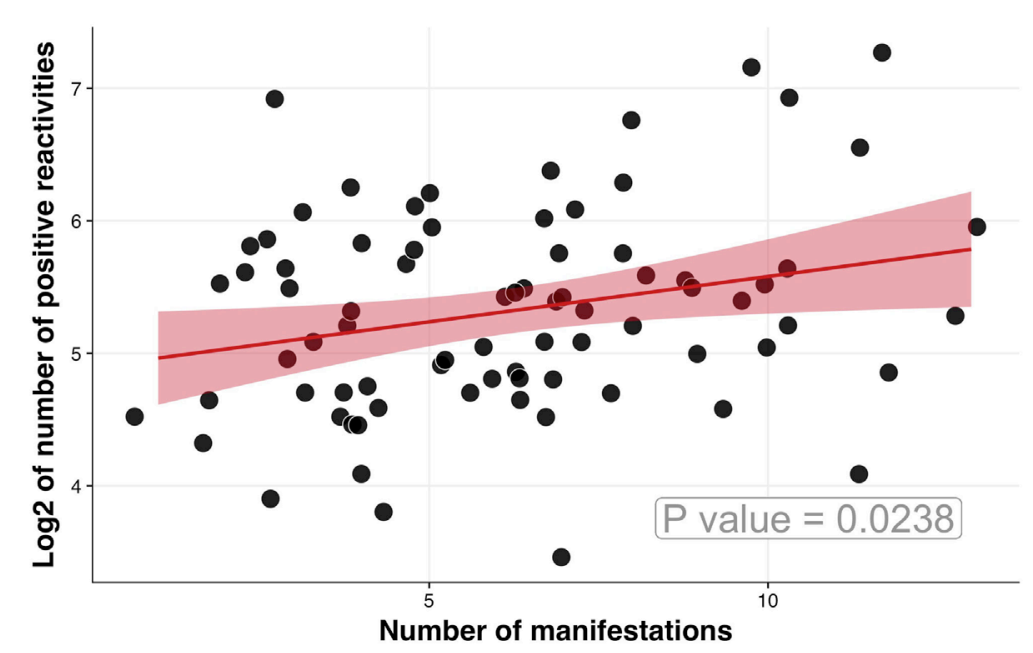

B

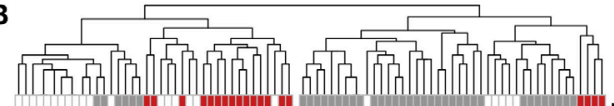

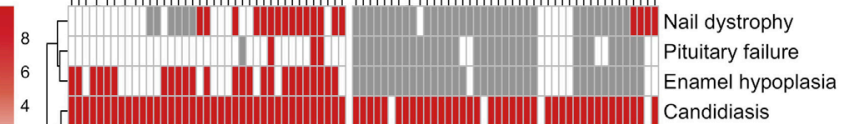

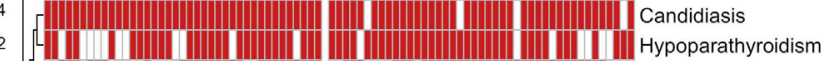

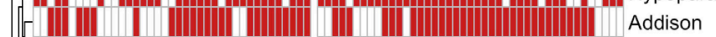

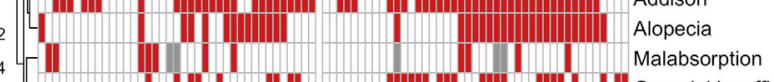

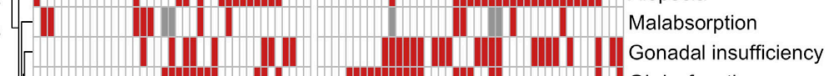

[E

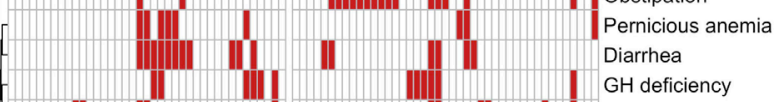

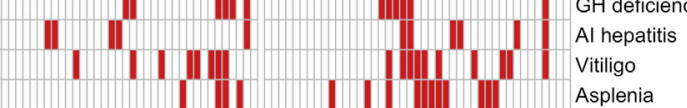

I || || || || || || || || $\mid \begin{aligned} & \text { Vitiligo } \\ & \text { Asplenia } \\ & \text { Type diabetes } \\ & \text { Tubulointerstitial }\end{aligned}$ II I I I I I I I I Inothyrorstitial nephritis | | | || | |||||||||||||||||||||||||||| Keratitis

E
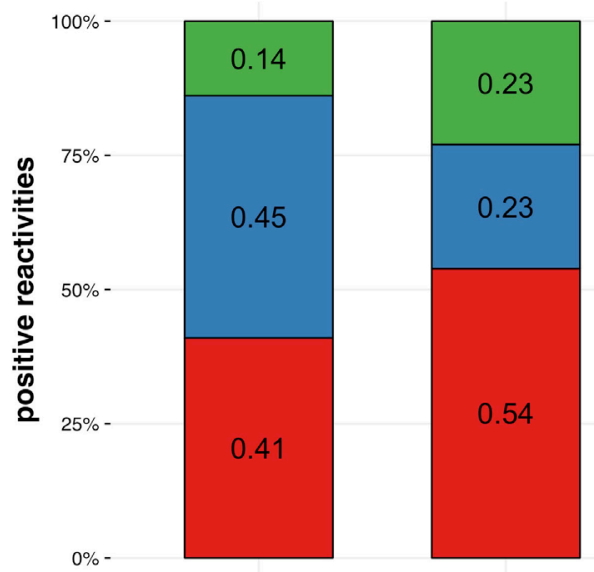

less than $10^{\prime}$ years apart more than 10 years apart 口Early पShared u Late

D

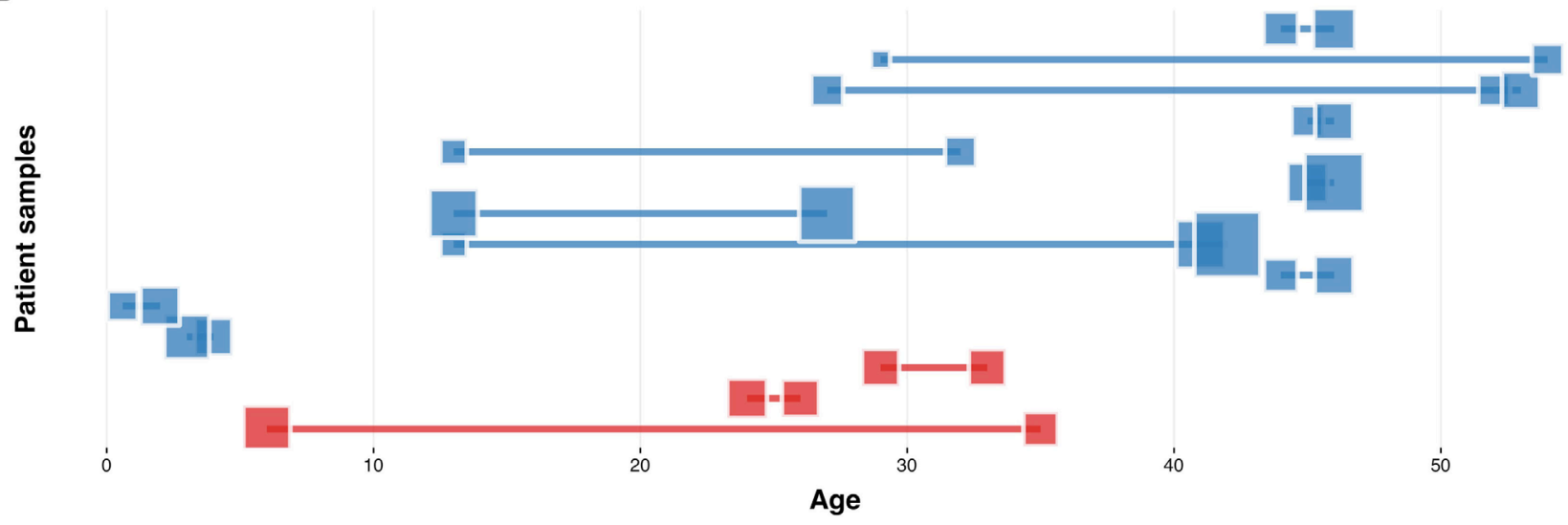

Trend Decreasing Increasing

FIGURE 4 | Continued 
FIGURE 4 | Continued

Characteristics of APECED patients and Protoarray reactivities. (A) Clustering patients and z-scores for the 50 most commonly recognized autoantigens using hierarchical clustering algorithm. (B) Clustering patients (columns) according to their clinical manifestations (rows). Red squares represent the presence of a given manifestation, while white and gray show absence of manifestation and missing information, respectively. (C) Positive correlation between the number of manifestations and the number of autoantigens ( $z>3$, logarithmized), correlation coefficient $\sim 0.29$. (D) Analysis of longitudinal serum samples taken at different time points. Blue squares and connecting lines $(n=11)$ correspond to samples with increased number of autoantigens and red squares and connecting lines $(n=3)$ correspond to samples with decreased or unchanged number of autoantigens. Each line and connected squares correspond to one APECED patient with samples collected at different time points. The locations of the squares on $y$-scale correspond to the age of the patient when the samples were collected. The sizes of the blue and red squares correspond to the number of positive autoantigens in corresponding samples. (E) The change of autoantigen profiles in samples collected longitudinally. The two columns show the autoantibody reactivities in patient samples taken less than 10 or more than 10 years apart. The autoantigens were compared between the samples of the same patient and divided into three categories according to their specificity: (i) specific to early sample, (ii) shared in early and late sample, and (iii) specific to late sample. The numbers in columns indicate the proportions of each category. Samples taken more than 10 years apart show higher percentage of autoantigens in the late samples, indicating that the autoantibody repertoire gets broader with time.
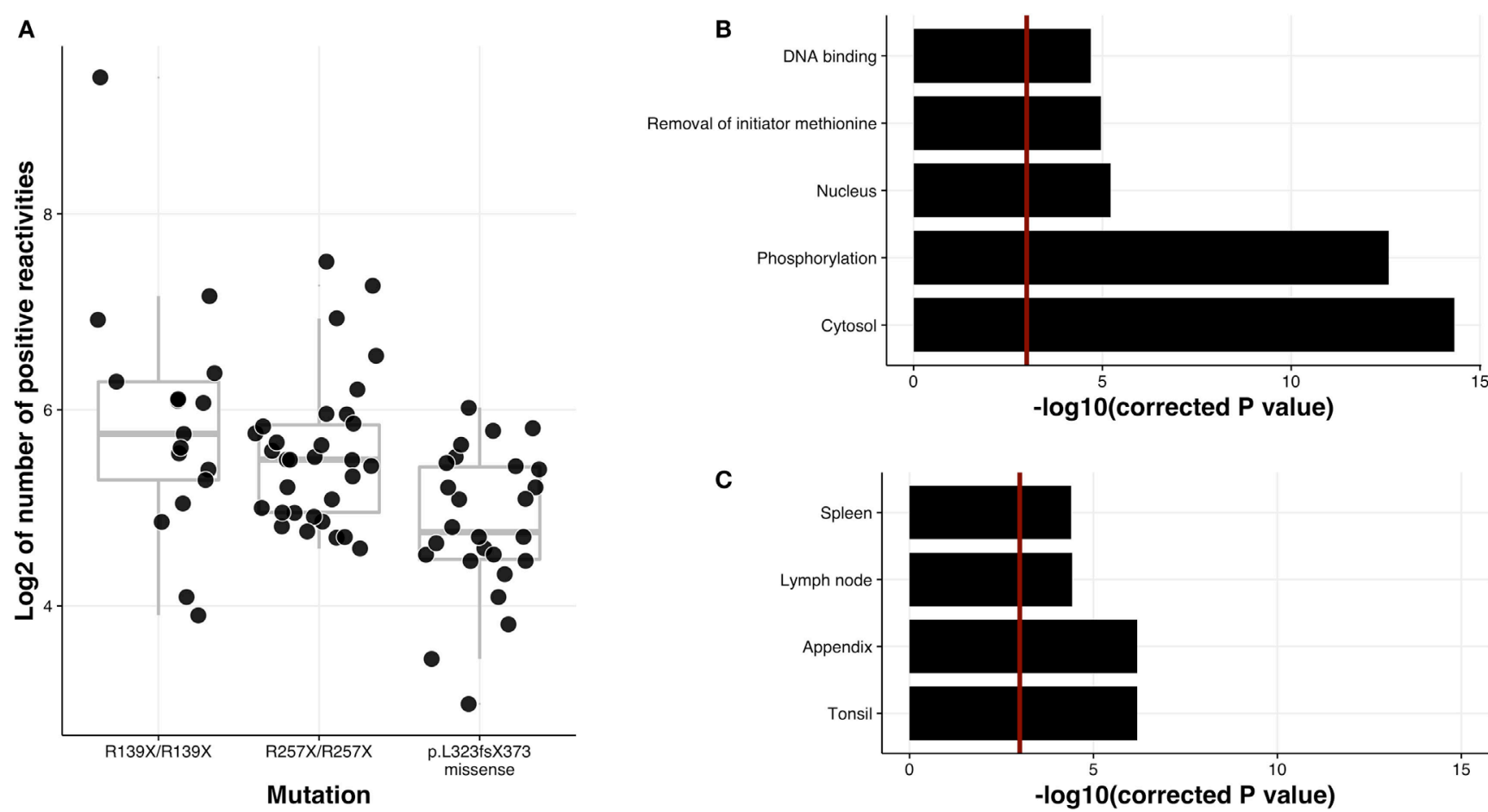

FIGURE 5 | Autoantibody correlation with APECED mutations and enrichment analysis for protein characteristics. (A) Correlation of the number of autoantibody targets with APECED mutations using pairwise comparisons with Tukey and Kramer (Nemenyi) test and Tukey-Dist approximation for independent samples. The first and the second mutation types are significantly different from the third type in terms of log2 of positive hits with $p$-values 0.0016 and 0.0072 , respectively. (B) Association of autoantigens with different protein characteristics. Length of each bar is directly proportional to the significance of the term, calculated with hypergeometric test. False discovery rate was used to correct for multiple testing. Red line is drawn at 0.05 significance level. (C) Association of positive reactivities with tissue-specific gene groups from Human Protein Atlas. Hypergeometric test was used to compute displayed $p$-values and false discovery rate was used to correct for multiple testing.

PSORT and YLoc databases and (ii) g:Profiler that searches for a cellular component as one of the Gene Ontology categories (27). The majority of autoantigens were significantly associated with intracellular location (adj. $p$-values $2.62 \mathrm{e}-07$ and $7.20 \mathrm{e}-03$ for Compartments and g:Profiler analysis, respectively), and in particular with either a cytosolic location (adj. $p$-values $6.04 \mathrm{e}-07$ and 3.01e-05 for Compartments and g:Profiler analysis, respectively) or, to a lesser extent, a nuclear location $(p=0.0054)$ (Table S2 in Supplementary Material).

Posttranslational modifications have been proposed to contribute to autoimmune responses (39). Indeed, when we compared our database with dbPTM datasets (22) comprising an integrated resource for protein posttranslational modifications, we found that the autoantigens were strongly enriched in proteins with histidine/serine/threonine phosphorylation (adj. $p=3.34 \mathrm{e}-06$ for HST). The association was significant even when threonine and serine phosphorylation were compared separately (adj. p-value 4.22e-06 for threonine only and $8.43 \mathrm{e}-04$ for serine only). However, tyrosine phosphorylation alone was not enriched and adding tyrosine phosphorylation as a category to the accumulated list of phosphorylated proteins decreased the significance level. We searched for the enrichment 

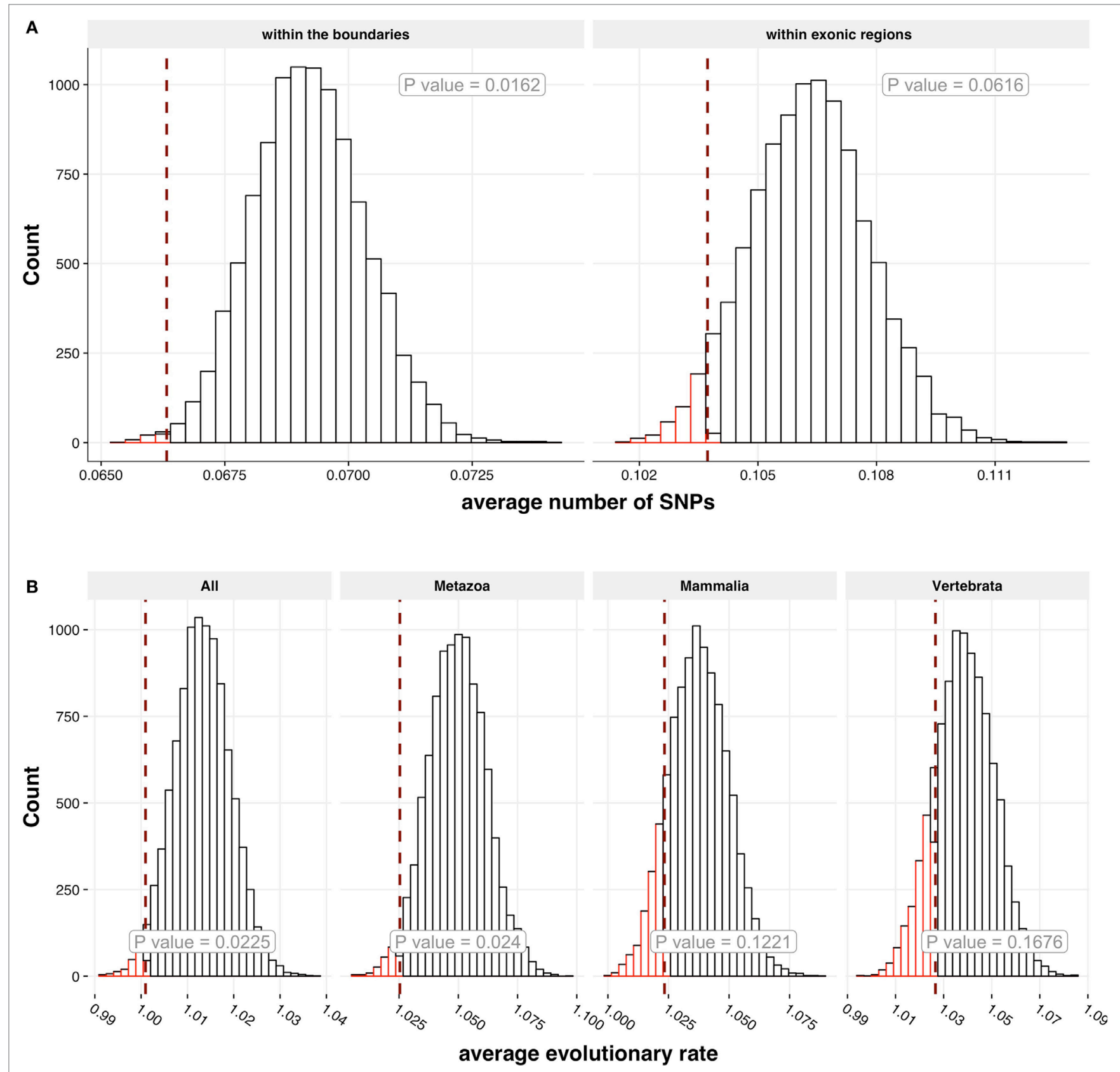

FIGURE 6 | Genetic polymorphisms and evolutionary conservation of APECED autoantigens. (A) Association of autoantigens with single nucleotide polymorphisms (SNPS). Two SNP categories were analyzed: the number of SNPs (i) within the boundaries of a gene (left histogram) and (ii) within exon regions of a gene (right histogram). For both histograms 10,000 randomly sampled groups of Protoarray proteins (genes) were generated with the size equal to the size of positive autoantigen group. On the $x$-axis the average number of SNPs is shown. The red dashed line shows the number of SNPs in positive autoantigen group. The $p$-value for each category of SNPs has been calculated in order to estimate the significance of a difference between randomly sampled groups and positive group. The autoantigen group has lower mean normalized SNP count, then it would be expected on average for the genes encoding proteins on Protoarray. (B) Evolutionary conservation rate. The four histograms that show distribution of average evolution rates for 10,000 randomly sampled groups of Protoarray genes were generated with the size equal to the size of positive autoantigen group. Each histogram represents a comparison of positive autoantigen group with ortholog genes in different evolutionary categories (all, metazoa, mammalia, or vertebrata species). On the $x$-axis the average evolutionary rate is shown. The red dashed line represents an average evolutionary rate of positive autoantigen group. Computed $p$-values indicate that autoantigens are on average more conserved in categories all and metazoa than the rest of the genes encoding proteins on Protoarray.

of specific protein features in UniProtKB and Superfamily databases comprising a collection of protein function information. This analysis surprisingly revealed that autoantigens were enriched for proteins from which the initiator methionine is processed by methionine aminopeptidases (adj. $p=0.0070$ ) and representation of proteins with DNA binding potential 
(adj. $p=0.0091$ ) (Supplementary Table S2 in Supplementary Material).

The genes encoding autoantigens have been suggested to be enriched for SNPs (40). However, when we studied SNP data from Ensembl.org, the genes encoding APECED autoantigens displayed fewer SNPs in their gene regions $(p=0.0126)$ as well as in exons $(p=0.0592)$ (Figure 6A). To study how evolutionary conserved the APECED autoantigen gene families (41) are, we used OrthoDB8 database to retrieve the calculated evolutionary rates of the autoantigen genes (as human counterparts) against ortholog genes in four different taxonomic levels (all species, metazoan, vertebrata, and mammalian species). We found that the genes encoding APECED autoantigens showed significant association with the two conserved taxonomic levels (all species, $p=0.0225$; metazoan species, $p=0.024$; and a similar trend in mammalian and vertebrata species categories) (Figure 6B) indicating an evolutionary conserved nature of APECED autoantigens. Thus, in comparison to all genes in the genome, APECED autoantigen genes have less genetic polymorphisms and are more conserved in evolution.

\section{Autoantigens Segregate Based on mTEC Expression and AIRE Dependence}

The key role of AIRE is considered to be the establishment of tolerance toward proteins restricted to discrete peripheral tissues, the so-called tissue-restricted (TR) antigens. However, unexpectedly, we did not find the APECED autoimmunome to be appreciably enriched in TR antigens. In fact, when each tissue was analyzed separately using the Human Protein Atlas database (20), the APECED autoantigens showed the strongest significant correlation with gene expression in lymphoid tissues, specifically tonsils, spleen, appendix, and lymph nodes (Figure 5C; Table S2 in Supplementary Material).

These findings notwithstanding, given that AIRE is the driving cause of APECED, we hypothesized that the autoantibody repertoire might target two components: TR antigens and non-tissue-restricted (NTR) antigens enriched in AIREindependent lymphoid tissue proteins. To test this hypothesis, we divided the genes encoding the autoantigens according to their TR or NTR expression pattern (Table S2 in Supplementary Material). We then cross-referenced mouse databases on the expression of genes by mature mTECs and to its subsets, including those where thymic AIRE expression is limited or lacking by knockout approach (42) (note the necessity to use mouse databases reflected by the lack of comprehensive human thymus gene expression data). Having converted human gene accession numbers to mouse counterparts, we found a very strong overlap of genes encoding APECED TR autoantigens with genes more highly expressed in mature mTEC compared to two Aire-negative subsets: immature mTEC $(p=0.00022)$ and Aire-negative mTEC subpopulation $(p=0.00016)$ (Figure 7). The genes downregulated in Aire-deficient mTEC cells were likewise enriched in genes encoding APECED TR autoantigens $(p=0.00022)$. By contrast, the NTR group showed no such correlations. Furthermore, the NTR group was associated even more strongly with intracellular phosphoproteins, expressed in lymphoid tissues.

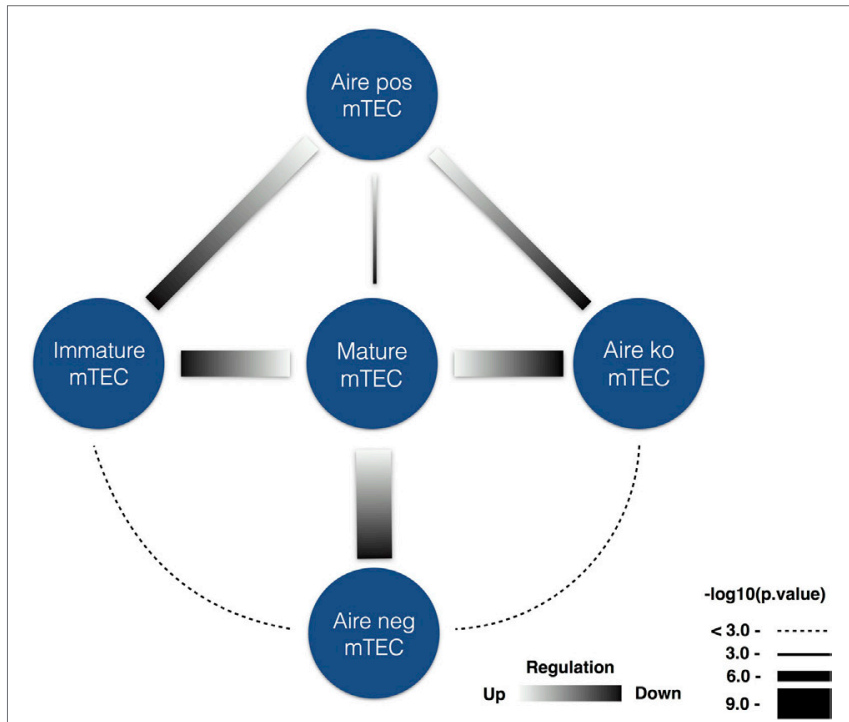

FIGURE 7 | Medullary thymic epithelial cells (mTEC) specific expression of tissue-restricted (TR) APECED autoantigens. TR APECED autoantigens but not the non-tissue-restricted (NTR) autoantigens were upregulated in mature Aire-expressing mTECs. Datalists [generated by Sansom et al. (42)] of differentially expressed genes in MTEC subsets were compared to the list of APECED autoantigens. Strong enrichment of TR autoantigens was found among differentially upregulated genes in mature mTEC subpopulation in comparison to (i) immature mTECs, (ii) Aire-negative mTECs, and (iii) Aire-knockout mTEC populations. Blue circles mark the corresponding cell populations, the gradient in connecting lines indicate whether the genes in comparison were up- or downregulated, and the width of the lines represents the logarithmized $p$-value. No significant overlap was found among NTR autoantigens differentially expressed in any of the studied thymic cell comparisons.

\section{DISCUSSION}

Our profiling of APECED autoantibody reactivities yielded a broad autoantibody repertoire and, except type I IFN and Th17 related cytokines, remarkable inter-individual variability. The number of candidate autoantigens ranged to almost thousand reactive proteins. Many of these proteins had lower signal intensities than type I IFN and Th17 cytokines and may partly represent a physiological (i.e., non-pathological) autoimmunity in the patients, similarly to natural autoantibodies in healthy individuals. However, in contrast to the genes activated by Aire in mTECs, only the minority was tissue-restricted proteins as we found many of the targets expressed in cell types with shared presence in lymphoid organs. Our study is in contrast to previous report (15), which identified only two novel targets (PDILT and MAGE-B2), also detected by our screening here, and with a key observation that autoimmunity in APECED preferentially targets molecules with restricted tissue expression profiles. The discrepancy may be related to conditions of serum samples, serum dilutions, differences in the versions of the Protoarray platforms, screening process and data analysis. While the previous study set highly stringent criteria considering only antigens that were common to the patient group we included the rare patient-specific reactivities, which to some extent may contain false positives. 
Among tissue-restricted proteins, we found reactivity to many CT-As, implying a central role of the thymus in antitumor immunity. These included members of MAGE-A, MAGE-B, and GAGE families, and in addition sperm-specific proteins, recognized by several (including female) patient sera. By contrast, we did not detect reactivities to MAGE-D or MAGE-E, the nonCT-A members of MAGE protein family (43) that are expressed in all tissues. Importantly, the only cell type expressing CT-As outside of tumors and testis is mTEC (44), and in agreement with our findings of extensive anti-CT-A immunity in APECED, Aire-deficient mice confer strong rejection of melanoma by thymus-dependent T cell responses (45-47). Furthermore, Aire has a dual role in the maintenance of immune tolerance as it also drives the development of a subset of regulatory T cells; indeed, suppressive Aire-dependent regulatory T cells were recruited to tumor sites in a mouse model of prostate cancer (48). Thus, our data in human patients highlight the role of AIRE in modulating immune responses to CT-As with implications for cancer immunotherapy.

The repertoire and properties of target autoantigens, which represent the collection of proteins without any recognizable rule, have been enigmatic. Clearly, the property to become an autoantigen is not a single intrinsic feature but a variable combination of protein characteristics. These features may include antigen structure, susceptibility to proteolytic cleavage, localization in apoptotic blebs, and release into the extracellular space (49). Other studies have highlighted structural motifs (50), evolutional conservation (41), posttranslational modifications (39), and a pro-inflammatory milieu of tissues (51). Post-translational modifications have been associated with various autoimmune diseases and include citrullination in rheumatoid arthritis (52), deamidation in celiac disease (53) and phosphorylation in systemic lupus erythematosus (54). We indeed found enrichment of evolutionarily conserved intracellular phosphoproteins, suggesting this posttranslational modification as one key factor in eliciting autoantibodies, and that specific protein properties contribute to B cell autoimmunity. In parallel to B cell epitopes, antigen phosphorylation is widespread and preserved among $\mathrm{T}$ cell epitopes on major histocompatibility complex (MHC) class I and II $(55,56)$, and deregulated phosphorylation creates tumor-specific neoantigens by affecting the antigenic identity or binding to MHC (57).

The high and neutralizing autoantibody titers to pro-inflammatory cytokines type I IFNs, IL-17A, IL-17F, and IL-22 implicate the inflammatory environment in the generation of the APECED autoantigen repertoire, although their scarcity in Aire-deficient mice (29) remains unexplained. Second, as high titer neutralizing anti-IFN $\alpha$ and anti-IFN $\omega$ autoantibodies are present in AIREdeficient thymoma and in recently described RAG-hypomorphic patients who lack AIRE expression (58), this strongly suggests the impairment of AIRE-dependent thymic tolerance in the development of anti-cytokine antibodies in humans. However, type I IFNs and Th17 cytokines are not restricted in their expression to mTECs; likewise the expression of GAD1, steroidogenic enzymes CYP17A1 and CYP21A2, and thyroglobulin is present in other thymic cells and they have no correlation with AIRE expression (59-61), in contrast to insulin and the alpha-subunit of AChR (59).
In AIRE-deficient thymoma samples, some APECED autoantigens are expectedly under-expressed, but many are not, including several adrenocortical, gonadal and neuro-ectodermal targets $(60,62)$. Hence, other cell types in the thymus should contribute to the negative selection of autoreactive $\mathrm{T}$ cells. Thus, other mechanisms should be considered, in addition to the role of AIRE in shaping negative selection by upregulating the expression of TR antigens. Indeed, the very early onset of clinical symptoms in APECED patients have suggested that AIRE deficiency may create an actively immunizing tissue environment where tolerance of AIRE-independent antigens is broken (63).

Despite the fact that approximately $90 \%$ of the overall group of autoantigens were not tissue-restricted, the correlation of TR-specific subset with mature mTEC population and Aire dependency was strikingly strong. Thus, our results demonstrate two distinct subgroups of autoantigens; first, the smaller subgroup, which consists of approximately $10 \%$ of autoantigens, is expressed in specific tissues, and of which many are expressed in mTECs under Aire regulation. The breakdown of tolerance to these APECED autoantigens is likely driven by their lack of expression in the thymus, causing the defect in negative selection of autoreactive thymocytes. The characteristic members of this group are GIF, CYP2A7, LCN1, as well as GAD2, the expression of which followed AIRE's expression pattern in thymomas (62), and conceivably CT-As, for which AIRE-dependency in human thymus remains unknown. By contrast, the second subgroup of NTR autoantigens is associated with intracellular phosphoproteins, expressed in multiple tissues with enrichment in lymphoid tissues (lymph node, spleen, bone marrow, and tonsils), and as such represents the mTEC-independent breach of tolerance, albeit caused by AIRE mutations. Autoantibodies to these proteins emerge by actions of yet unknown mechanisms associated, for example, with apoptotic cell death or by dysfunctional B cell tolerance during differentiation in lymphoid tissues. Our findings are supported by RNA-seq analysis of autoantigen-encoding genes, which identified a subset of autoantigens, associated with autoimmune diseases, to be expressed ubiquitously but enriched in immune tissues (64). It should be noted that, in contrast to other immune tissues, the thymus was not available via Human Protein Atlas (20). Nevertheless, given the proposed hypothesis of active autoimmunization in AIRE-deficient thymus, this raises intriguing questions of whether the second set of lymphoid autoantigens might represent the antigens acquired form apoptotic thymocytes.

Finally, we identified so far unrevealed clinical associations with pernicious anemia, vitiligo, and autoimmune hepatitis. These correlations need further studies as well as the analysis of the expression of target proteins in corresponding diseased organs and tissues. Despite several outstanding correlations of clinical disease and specific autoantibodies in APECED, the majority of autoantibody reactivities seem not to have relevance to specific clinical entities. They can be just a bystander result of $\mathrm{T}$ cell responses, not necessarily reflecting full-blown autoimmune attack or their reactivity may depend on other factors, for example, the presence of posttranslational modifications or complexes with nucleic acids, which can operate as adjuvants. In contrast to single autoantigen responses, our results highlight 
the overall spread of autoantibody repertoire as a driver for the expansion of clinical profiles in APECED. Alternatively, these autoantibodies may have unexpected protective roles as we recently showed a negative correlation of anti-IFNA antibodies with the incidence of T1D (9). The unexpectedly broad APECED autoimmunome forms a unique platform for further analysis of B cell autoimmunity toward self-antigens and their correlation with clinical manifestations.

\section{ETHICS STATEMENT}

The study was carried out in accordance with the recommendations of local ethics committees (Finland: HUS Medical ERB, 8/13/03/01/2009; Slovenia: National Medical Ethics Committee number 22/09/09 and 28/02/13; Italy: Ethics Committee Prot. PG/2015/20440; Norway: Research Ethics Committee of Western Norway, health registry number 047.96, bio-bank number 2013-1504, project number 2012/1850; Estonia: Research Ethics Committee of the University of Tartu, 235/M-23) with written informed consent from all subjects. All subjects gave written informed consent in accordance with the Declaration of Helsinki.

\section{AUTHOR CONTRIBUTIONS}

DF, KKisand, $\mathrm{CH}$, and PP analyzed the Protoarray data. $\mathrm{CH}$ and MR assisted and supervised seroreactivity screenings of

\section{REFERENCES}

1. Goodnow CC, Vinuesa CG, Randall KL, Mackay F, Brink R. Control systems and decision making for antibody production. Nat Immunol (2010) 11(8):681-8. doi:10.1038/ni.1900

2. Kisand K, Peterson P. Autoimmune polyendocrinopathy candidiasis ectodermal dystrophy. J Clin Immunol (2015) 35(5):463-78. doi:10.1007/ s10875-015-0176-y

3. Abramson J, Husebye ES. Autoimmune regulator and self-tolerance - molecular and clinical aspects. Immunol Rev (2016) 271(1):127-40. doi:10.1111/ imr.12419

4. Mathis D, Benoist C. Aire. Annu Rev Immunol (2009) 27:287-312. doi:10.1146/ annurev.immunol.25.022106.141532

5. Peterson P, Org T, Rebane A. Transcriptional regulation by AIRE: molecular mechanisms of central tolerance. Nat Rev Immunol (2008) 8(12):948-57. doi: $10.1038 /$ nri2450

6. Meager A, Visvalingam K, Peterson P, Moll K, Murumagi A, Krohn K, et al. Anti-interferon autoantibodies in autoimmune polyendocrinopathy syndrome type 1. PLoS Med (2006) 3(7):e289. doi:10.1371/journal.pmed.0030289

7. Wolff ASB, Sarkadi AK, Marodi L, Kaerner J, Orlova E, Oftedal BEV, et al. Anti-cytokine autoantibodies preceding onset of autoimmune polyendocrine syndrome type I features in early childhood. J Clin Immunol (2013) 33(8):1341-8. doi:10.1007/s10875-013-9938-6

8. Kisand K, Link M, Wolff AS, Meager A, Tserel L, Org T, et al. Interferon autoantibodies associated with AIRE deficiency decrease the expression of IFN-stimulated genes. Blood (2008) 112(7):2657-66. doi:10.1182/ blood-2008-03-144634

9. Meyer S, Woodward M, Hertel C, Vlaicu P, Haque Y, Kärner J, et al. AIREdeficient patients harbor unique high-affinity disease-ameliorating autoantibodies. Cell (2016) 166(3):582-95. doi:10.1016/j.cell.2016.06.024

10. Kisand K, Boe Wolff AS, Podkrajsek KT, Tserel L, Link M, Kisand KV, et al. Chronic mucocutaneous candidiasis in APECED or thymoma patients correlates with autoimmunity to Th17-associated cytokines. J Exp Med (2010) 207(2):299-308. doi:10.1084/jem.20091669
Protoarrays. MP and ARemm performed LIPS analyses. PA, HP, and JV contributed and supervised bioinformatic analyses of Protoarray data. AP, AM, KP, TB, ØB, AW, EH, NK, KKrohn, and ARanki sampled APECED patient samples and contributed to clinical database. AH and PP supervised research. DF, KKisand, $\mathrm{CH}, \mathrm{AH}$, and $\mathrm{PP}$ wrote the paper with contributions from other authors.

\section{ACKNOWLEDGMENTS}

We are extremely grateful to APECED patients who provided sera and to the Finnish APECED patient association, attending physicians and care-takers of the patients. We are likewise grateful to ImmunoQure AG and Drs. Ed Stuart and Karsten Henco for their help and discussions of this research. We thank financial support from Wellcome Trust grant 106292/Z/14/Z, Cancer Research UK, Estonian Research Council grant IUT2-2 and IUT34-4. The research was also supported by the European Union through the European Regional Development Fund (Project No. 20142020.4.01.15-0012) and CoE of Estonian ICT research EXCITE.

\section{SUPPLEMENTARY MATERIAL}

The Supplementary Material for this article can be found online at http://journal.frontiersin.org/article/10.3389/fimmu.2017.00976/ full\#supplementary-material.

11. Puel A, Doffinger R, Natividad A, Chrabieh M, Barcenas-Morales G, Picard C, et al. Autoantibodies against IL-17A, IL-17F, and IL-22 in patients with chronic mucocutaneous candidiasis and autoimmune polyendocrine syndrome type I. J Exp Med (2010) 207(2):291-7. doi:10.1084/jem.20091983

12. Meffre E, Wardemann H. B-cell tolerance checkpoints in health and autoimmunity. Curr Opin Immunol (2008) 20(6):632-8. doi:10.1016/j.coi.2008.09.001

13. Herold KC, Vignali DA, Cooke A, Bluestone JA. Type 1 diabetes: translating mechanistic observations into effective clinical outcomes. Nat Rev Immunol (2013) 13(4):243-56. doi:10.1038/nri3422

14. Bratland E, Husebye ES. Cellular immunity and immunopathology in autoimmune Addison's disease. Mol Cell Endocrinol (2011) 336(1-2):180-90. doi:10.1016/j.mce.2010.12.015

15. Landegren N, Sharon D, Freyhult E, Hallgren Å, Eriksson D, Edqvist PH, et al. Proteome-wide survey of the autoimmune target repertoire in autoimmune polyendocrine syndrome type 1. Sci Rep (2016) 6:20104. doi:10.1038/ srep20104

16. Abel L, Kutschki S, Turewicz M, Eisenacher M, Stoutjesdijk J, Meyer HE, et al. Autoimmune profiling with protein microarrays in clinical applications. Biochim Biophys Acta (2014) 1844(5):977-87. doi:10.1016/j. bbapap.2014.02.023

17. Sboner A, Karpikov A, Chen G, Smith M, Mattoon D, Dawn M, et al. Robustlinear-model normalization to reduce technical variability in functional protein microarrays. JProteome Res (2009) 8(12):5451-64. doi:10.1021/ pr900412k

18. Turewicz M, May C, Ahrens M, Woitalla D, Gold R, Casjens S, et al. Improving the default data analysis workflow for large autoimmune biomarker discovery studies with ProtoArrays. Proteomics (2013) 13(14):2083-7. doi:10.1002/ pmic. 201200518

19. Ritchie ME, Phipson B, Wu D, Hu Y, Law CW, Shi W, et al. limma powers differential expression analyses for RNA-sequencing and microarray studies. Nucleic Acids Res (2015) 43(7):e47. doi:10.1093/nar/gkv007

20. Uhlén M, Fagerberg L, Hallström BM, Lindskog C, Oksvold P, Mardinoglu A, et al. Proteomics. Tissue-based map of the human proteome. Science (2015) 347(6220):1260419. doi:10.1126/science. 1260419 
21. Binder JX, Pletscher-FrankildS, Tsafou K, Stolte C, O’DonoghueSI,Schneider R, et al. COMPARTMENTS: unification and visualization of protein subcellular localization evidence. Database (Oxford) (2014) 2014:bau012. doi:10.1093/ database/bau012

22. Lee TY, Huang HD, Hung JH, Huang HY, Yang YS, Wang TH. dbPTM: an information repository of protein post-translational modification. Nucleic Acids Res (2006) 34(Database issue):D622-7. doi:10.1093/nar/ gkj083

23. Consortium U. UniProt: a hub for protein information. Nucleic Acids Res (2015) 43(Database issue):D204-12. doi:10.1093/nar/gku989

24. Yates A, Akanni W, Amode MR, Barrell D, Billis K, Carvalho-Silva D, et al. Ensembl 2016. Nucleic Acids Res (2016) 44(D1):D710-6. doi:10.1093/nar/ gkv1157

25. KriventsevaEV,TegenfeldtF,PettyTJ,WaterhouseRM,SimãoFA,PozdnyakovIA, et al. OrthoDB v8: update of the hierarchical catalog of orthologs and the underlying free software. Nucleic Acids Res (2015) 43(Database issue):D250-6. doi:10.1093/nar/gku1220

26. Gough J, Karplus K, Hughey R, Chothia C. Assignment of homology to genome sequences using a library of hidden Markov models that represent all proteins of known structure. J Mol Biol (2001) 313(4):903-19. doi:10.1006/ jmbi.2001.5080

27. Reimand J, Arak T, Adler P, Kolberg L, Reisberg S, Peterson H, et al. g:Profiler-a web server for functional interpretation of gene lists (2016 update). Nucleic Acids Res (2016) 44(W1):W83-9. doi:10.1093/nar/gkw199

28. Burbelo PD, Browne SK, Sampaio EP, Giaccone G, Zaman R, Kristosturyan E, et al. Anti-cytokine autoantibodies are associated with opportunistic infection in patients with thymic neoplasia. Blood (2010) 116(23):4848-58. doi:10.1182/ blood-2010-05-286161

29. Kärner J, Meager A, Laan M, Maslovskaja J, Pihlap M, Remm A, et al. Anticytokine autoantibodies suggest pathogenetic links with autoimmune regulator deficiency in humans and mice. Clin Exp Immunol (2013) 171(3):263-72. doi:10.1111/cei.12024

30. Navone R, Lunardi C, Gerli R, Tinazzi E, Peterlana D, Bason C, et al. Identification of tear lipocalin as a novel autoantigen target in Sjögren's syndrome. J Autoimmun (2005) 25(3):229-34. doi:10.1016/j.jaut.2005. 09.021

31. Pisetsky DS. The complex role of DNA, histones and HMGB1 in the pathogenesis of SLE. Autoimmunity (2014) 47(8):487-93. doi:10.3109/08916934.2 014.921811

32. Simpson AJ, Caballero OL, Jungbluth A, Chen YT, Old LJ. Cancer/testis antigens, gametogenesis and cancer. Nat Rev Cancer (2005) 5(8):615-25. doi: $10.1038 / \mathrm{nrc} 1669$

33. Zhang XD, Miao SY, Wang LF, Li Y, Zong SD, Yan YC, et al. Human sperm membrane protein (hSMP-1): a developmental testis-specific component during germ cell differentiation. Arch Androl (2000) 45(3):239-46. doi:10.1080/01485010050194020

34. de Bock L, Somers K, Fraussen J, Hendriks JJ, van Horssen J, Rouwette M, et al. Sperm-associated antigen 16 is a novel target of the humoral autoimmune response in multiple sclerosis. J Immunol (2014) 193(5):2147-56. doi:10.4049/ jimmunol.1401166

35. Toh BH. Pathophysiology and laboratory diagnosis of pernicious anemia. Immunol Res (2017) 65:326-30. doi:10.1007/s12026-016-8841-7

36. Gebre-Medhin G, Husebye ES, Gustafsson J, Winqvist O, Goksoyr A, Rorsman F, et al. Cytochrome P450IA2 and aromatic L-amino acid decarboxylase are hepatic autoantigens in autoimmune polyendocrine syndrome type I. FEBS Lett (1997) 412(3):439-45. doi:10.1016/S0014-5793(97)00797-7

37. Husebye ES, Gebre-Medhin G, Tuomi T, Perheentupa J, Landin-Olsson M, Gustafsson J, et al. Autoantibodies against aromatic L-amino acid decarboxylase in autoimmune polyendocrine syndrome type I. J Clin Endocrinol Metab (1997) 82(1):147-50. doi:10.1210/jcem.82.1.3647

38. Landegren N, Sharon D, Shum AK, Khan IS, Fasano KJ, Hallgren A, et al. Transglutaminase 4 as a prostate autoantigen in male subfertility. Sci Transl Med (2015) 7(292):292ra101. doi:10.1126/scitranslmed.aaa9186

39. Doyle HA, Yang ML, Raycroft MT, Gee RJ, Mamula MJ. Autoantigens: novel forms and presentation to the immune system. Autoimmunity (2014) 47(4):220-33. doi:10.3109/08916934.2013.850495

40. Stadler MB, Arnold D, Frieden S, Luginbühl S, Stadler BM. Single nucleotide polymorphisms as a prerequisite for autoantigens. Eur J Immunol (2005) 35(2):371-8. doi:10.1002/eji.200425481
41. Backes C, Ludwig N, Leidinger P, Harz C, Hoffmann J, Keller A, et al. Immunogenicity of autoantigens. BMC Genomics (2011) 12:340. doi:10.1186/1471-2164-12-340

42. Sansom SN, Shikama-Dorn N, Zhanybekova S, Nusspaumer G, Macaulay IC, Deadman ME, et al. Population and single-cell genomics reveal the Aire dependency, relief from Polycomb silencing, and distribution of self-antigen expression in thymic epithelia. Genome Res (2014) 24(12): 1918-31. doi:10.1101/gr.171645.113

43. Katsura Y, Satta Y. Evolutionary history of the cancer immunity antigen MAGE gene family. PLoS One (2011) 6(6):e20365. doi:10.1371/journal.pone.0020365

44. Gotter J, Brors B, Hergenhahn M, Kyewski B. Medullary epithelial cells of the human thymus express a highly diverse selection of tissue-specific genes colocalized in chromosomal clusters. JExp Med (2004) 199(2):155-66. doi:10.1084/jem.20031677

45. Träger U, Sierro S, Djordjevic G, Bouzo B, Khandwala S, Meloni A, et al. The immune response to melanoma is limited by thymic selection of self-antigens. PLoS One (2012) 7(4):e35005. doi:10.1371/journal.pone.0035005

46. Zhu ML, Nagavalli A, Su MA. Aire deficiency promotes TRP-1-specific immune rejection of melanoma. Cancer Res (2013) 73(7):2104-16. doi:10.1158/0008-5472.CAN-12-3781

47. Khan IS, Mouchess ML, Zhu ML, Conley B, Fasano KJ, Hou Y, et al. Enhancement of an anti-tumor immune response by transient blockade of central T cell tolerance. J Exp Med (2014) 211(5):761-8. doi:10.1084/ jem.20131889

48. Malchow S, Leventhal DS, Nishi S, Fischer BI, Shen L, Paner GP, et al. Aire-dependent thymic development of tumor-associated regulatory $\mathrm{T}$ cells. Science (2013) 339(6124):1219-24. doi:10.1126/science.1233913

49. Plotz PH. The autoantibody repertoire: searching for order. Nat Rev Immunol (2003) 3(1):73-8. doi:10.1038/nri976

50. Carl PL, Temple BR, Cohen PL. Most nuclear systemic autoantigens are extremely disordered proteins: implications for the etiology of systemic autoimmunity. Arthritis Res Ther (2005) 7(6):R1360-74. doi:10.1186/ar1832

51. Suurmond J, Diamond B. Autoantibodies in systemic autoimmune diseases: specificity and pathogenicity. J Clin Invest (2015) 125(6):2194-202. doi:10.1172/ JCI78084

52. Clancy KW, Weerapana E, Thompson PR. Detection and identification of protein citrullination in complex biological systems. Curr Opin Chem Biol (2016) 30:1-6. doi:10.1016/j.cbpa.2015.10.014

53. Stamnaes J, Sollid LM. Celiac disease: autoimmunity in response to food antigen. Semin Immunol (2015) 27(5):343-52. doi:10.1016/j.smim.2015. 11.001

54. Neugebauer KM, Merrill JT, Wener MH, Lahita RG, Roth MB. SR proteins are autoantigens in patients with systemic lupus erythematosus. Importance of phosphoepitopes. Arthritis Rheum (2000) 43(8):1768-78. doi:10.1002/1529-0131(200008)43:8<1768:AID-ANR13>3.0.CO;2-9

55. Depontieu FR, Qian J, Zarling AL, McMiller TL, Salay TM, Norris A, et al. Identification of tumor-associated, MHC class II-restricted phosphopeptides as targets for immunotherapy. Proc Natl Acad Sci U S A (2009) 106(29): 12073-8. doi:10.1073/pnas.0903852106

56. Zarling AL, Polefrone JM, Evans AM, Mikesh LM, Shabanowitz J, Lewis ST, et al. Identification of class I MHC-associated phosphopeptides as targets for cancer immunotherapy. Proc Natl Acad Sci U S A (2006) 103(40):14889-94. doi:10.1073/pnas.0604045103

57. Mohammed F, Cobbold M, Zarling AL, Salim M, Barrett-Wilt GA, Shabanowitz J, et al. Phosphorylation-dependent interaction between antigenic peptides and MHC class I: a molecular basis for the presentation of transformed self. Nat Immunol (2008) 9(11):1236-43. doi:10.1038/ ni. 1660

58. Walter JE, Rosen LB, Csomos K, Rosenberg JM, Mathew D, Keszei M, et al. Broad-spectrum antibodies against self-antigens and cytokines in RAG deficiency. J Clin Invest (2015) 125(11):4135-48. doi:10.1172/JCI80477

59. Taubert R, Schwendemann J, Kyewski B. Highly variable expression of tissue-restricted self-antigens in human thymus: implications for selftolerance and autoimmunity. Eur J Immunol (2007) 37(3):838-48. doi:10.1002/ eji.200636962

60. Ströbel P, Murumägi A, Klein R, Luster M, Lahti M, Krohn K, et al. Deficiency of the autoimmune regulator AIRE in thymomas is insufficient to elicit autoimmune polyendocrinopathy syndrome type 1 (APS-1). J Pathol (2007) 211(5):563-71. doi:10.1002/path.2141 
61. Li B, Li J, Hsieh CS, Hale LP, Li YJ, Devlin BH, et al. Characterization of cultured thymus tissue used for transplantation with emphasis on promiscuous expression of thyroid tissue-specific genes. Immunol Res (2009) 44(1-3):71-83. doi:10.1007/s12026-008-8083-4

62. Wolff AS, Kärner J, Owe JF, Oftedal BE, Gilhus NE, Erichsen MM, et al. Clinical and serologic parallels to APS-I in patients with thymomas and autoantigen transcripts in their tumors. JImmunol (2014) 193(8):3880-90. doi:10.4049/ jimmunol.1401068

63. Kisand K, Lilic D, Casanova JL, Peterson P, Meager A, Willcox N. Mucocutaneous candidiasis and autoimmunity against cytokines in APECED and thymoma patients: clinical and pathogenetic implications. Eur J Immunol (2011) 41(6):1517-27. doi:10.1002/eji.201041253

64. Burbelo PD, Iadarola MJ, Alevizos I, Sapio MR. Transcriptomic segregation of human autoantigens useful for the diagnosis of autoimmune diseases. Mol Diagn Ther (2016) 20(5):415-27. doi:10.1007/s40291-016-0211-6
Conflict of Interest Statement: The authors $\mathrm{CH}$ and MR were the employees of Immunoqure AG when the study was conducted. PP, KKisand, KKrohn, AR, and $\mathrm{AH}$ are shareholders of ImmunoQure AG. The remaining authors declare no competing financial interests. The work is relevant US Patent Application US20170051055A1 (Human anti-IFN-alpha antibodies).

Copyright (c) 2017 Fishman, Kisand, Hertel, Rothe, Remm, Pihlap, Adler, Vilo, Peet, Meloni, Podkrajsek, Battelino, Bruserud, Wolff, Husebye, Kluger, Krohn, Ranki, Peterson, Hayday and Peterson. This is an open-access article distributed under the terms of the Creative Commons Attribution License (CC BY). The use, distribution or reproduction in other forums is permitted, provided the original author(s) or licensor are credited and that the original publication in this journal is cited, in accordance with accepted academic practice. No use, distribution or reproduction is permitted which does not comply with these terms. 